\title{
On a certain problem of Ulam and its generalization
}

\author{
Anup Biswas \\ Anup Biswas received his Bachelor of Science degree at the University of Calcutta, \\ India, in 2005. He was awarded his Master degree from the Tata Institute of Funda- \\ mental Research in Bangalore in 2007, where he is currently employed as a research \\ scholar.
}

\section{Introduction}

Let $\mathbb{N}$ be the set of all positive integers with usual operations $a+b \equiv s(a, b)$ (addition) and $a * b \equiv m(a, b)$ (multiplication). For every bijection $p: \mathbb{N} \times \mathbb{N} \rightarrow \mathbb{N}$ define two mappings $\sigma$ and $\mu$ from $\mathbb{N}$ to $\mathbb{N}$ such that $\sigma(c)=\sigma(p(a, b))=s(a, b)$ and $\mu(c)=$ $\mu(p(a, b))=m(a, b)$ for all $c \in \mathbb{N}$. Such a bijection $p$ is known as Peano mapping. In [1, p. 32] S.M. Ulam asked "Does there exist a Peano mapping $p$ such that addition commutes with multiplication in the sense that $\sigma(\mu(c))=\mu(\sigma(c))$ for all $c \in \mathbb{N}$ ?". No examples were known, and then the well-known Peano mapping $p(a, b)=2^{a-1}(2 b-1)$ was seen by Ulam not to work, as $\mu(\sigma(2))=\mu(3)=2, \sigma(\mu(2))=\sigma(2)=3$. Though the question is very natural and fascinating, it never got an answer since then.

In this article, we answer Ulam's question in showing that no Peano mapping exists with the desired property. The proof is simple and totally elementary.

In dem nachfolgenden Beitrag wird ein auf S.M. Ulam zurückgehendes Problem gelöst. Ist $p: \mathbb{N} \times \mathbb{N} \rightarrow \mathbb{N}$ eine Bijektion, eine sog. Peano-Abbildung, und bedeuten $s, m: \mathbb{N} \times \mathbb{N} \rightarrow \mathbb{N}$ die Abbildungen, die durch $s(a, b)=a+b$ (Addition) bzw. $m(a, b)=a \cdot b$ (Multiplikation) gegeben sind, so seien $\sigma, \mu: \mathbb{N} \rightarrow \mathbb{N}$ durch $\sigma=s \circ p^{-1}$ bzw. $\mu=m \circ p^{-1}$ festgelegt. Es besteht nun die Frage, ob es eine Peano-Abbildung $p$ mit der Eigenschaft gibt, dass $\sigma$ und $\mu$ vertauschen, d.h. dass für alle natürlichen Zahlen $c$ die Gleichheit $\sigma(\mu(c))=\mu(\sigma(c))$ gilt. Die Abbildung $p$, gegeben durch $p(a, b)=2^{a-1}(2 b-1)$, ist beispielsweise eine Peano-Abbildung; allerdings gilt in diesem Fall $\sigma(\mu(2))=3$ und $\mu(\sigma(2))=2$. Der Autor zeigt, dass dieses Beispiel keine Ausnahme darstellt, dass es also keine Peano-Abbildung gibt, die das gewünschte Vertauschen von $\sigma$ und $\mu$ zulässt. 
We further show that no such Peano mapping exists in $\mathbb{R}^{+}$. The proof uses only quadratic extensions of $\mathbb{Q}$, and so works as well for the algebraic numbers $\mathbb{A}^{+}$, but it leaves the case of $\mathbb{Q}^{+}$open.

\section{Proof for $\mathbb{N}$}

Let us assume that there exists a Peano map $p$ such that $\sigma$ and $\mu$ commute. Note that $\sigma=s \circ p^{-1}$ and $\mu=m \circ p^{-1}$. Therefore we have

$$
m \circ p^{-1} \circ s \circ p^{-1}=s \circ p^{-1} \circ m \circ p^{-1},
$$

or

$$
m \circ p^{-1} \circ s=s \circ p^{-1} \circ m .
$$

To simplify notation, write $f: \mathbb{N} \rightarrow \mathbb{N} \times \mathbb{N}$ for the bijection $p^{-1}$. Then

$$
(m \circ f \circ s)(a, b)=(s \circ f \circ m)(a, b) \quad \forall(a, b) \in \mathbb{N} \times \mathbb{N} .
$$

We first show that $f(1)=(1,1)$. Assume to the contrary that there exists an integer $k>1$ such that $f(k)=(1,1)$. So

$(m \circ f \circ s)(k-1,1)=m(f(k))=m(1,1)=1=(s \circ f \circ m)(k-1,1)=s(f(k-1))$.

This is impossible, as $s(f(n)) \geq 2$ in $\mathbb{N}$. Moreover $s(f(n))=2$ implies $n=1$.

As a consequence,

$$
(s \circ f \circ m)(1,1)=s(f(1))=2=(m \circ f \circ s)(1,1)=m(f(2)) .
$$

Hence $f(2) \in\{(1,2),(2,1)\}$. If $f(2)=(1,2)$, there exists an integer $k>2$ such that $f(k)=(2,1)$. Again from (1) we have

$$
(m \circ f \circ s)(k-1,1)=2=(s \circ f \circ m)(k-1,1)=s(f(k-1)) .
$$

So $k-1=1$ or $k=2$, a contradiction. The same argument works with $f(2)=(1,2)$. This proves that there is no Peano mapping for which $\sigma$ and $\mu$ commute.

\section{Proof for $\mathbb{R}^{+}$}

As above, suppose to the contrary that a bijection $f: \mathbb{R}^{+} \rightarrow \mathbb{R}^{+} \times \mathbb{R}^{+}$exists, satisfying

$$
(m \circ f \circ s)(a, b)=(s \circ f \circ m)(a, b) \quad \forall(a, b) \in \mathbb{R}^{+} \times \mathbb{R}^{+} .
$$

Take three real numbers $u, v, w$ such that the system of five equations

$$
x_{i}+y_{i}=u_{i}, \quad x_{i} \cdot y_{i}=v_{i} \quad(1 \leq i \leq 5)
$$


with

$$
\begin{aligned}
u_{1}=u_{2}=u_{4}=v_{1}=v_{3} & =v_{5}=u, \\
u_{3} & =v_{2}=v, \\
u_{5} & =v_{4}=w
\end{aligned}
$$

has positive real solutions (e.g. $u=6, v=7, w=8$ ).

Let $f(u)=(a, b)$. From $m \circ f\left(x_{i}+y_{i}\right)=s \circ f\left(x_{i} \cdot y_{i}\right)$ (equation (2)), one gets

$$
\begin{aligned}
m \circ f(u) & =m \circ f(v)=m \circ f(w)=a b, \\
s \circ f(u) & =s \circ f(v)=s \circ f(w)=a+b .
\end{aligned}
$$

As there are at most two solutions for $f(u), f(v)$, and $f(w)$, namely $(a, b)$ and $(b, a)$, the map $f$ cannot be a bijection, establishing the desired result.

\section{References}

[1] Ulam, S.M.: Problems in Modern Mathematics. John Wiley, New York 1964.

\section{Anup Biswas}

Centre for Applicable Mathematics

Tata Institute of Fundamental Research

Post Bag No 6503, Chikkabommasandra

Bangalore-560065, India

e-mail: anup@math.tifrbng.res.in 